\title{
Fractional Ostrowski type inequalities for bounded functions
}

\author{
Samet Erden' ${ }^{1}$, Hüseyin Budak², Mehmet Zeki Sarikaya², Sabah Iftikhar ${ }^{3}$ and Poom Kumam³,4*
}

"Correspondence:

poom.kum@kmutt.ac.th

${ }^{3}$ Center of Excellence in Theoretical

and Computational Science

(TaCS-CoE), Science Laboratory

Building, Faculty of Science, King

Mongkut's University of Technology

Thonburi (KMUTT), Bangkok,

Thailand

${ }^{4}$ KMUTT Fixed Point Research Laboratory, KMUTT-Fixed Point

Theory and Applications Research

Group, SCL 802 Fixed Point

Laboratory, Department of

Mathematics, Faculty of Science,

King Mongkut's University of

Technology Thonburi (KMUTT),

Bangkok, Thailand

Full list of author information is

available at the end of the article

\begin{abstract}
We first establish some results involving Riemann-Liouville fractional integrals for partially differentiable functions. Then we obtain some fractional Ostrowski type inequalities for functions in class of functions $L_{p}, L_{\infty}$ and $L_{1}$, respectively. We also give some midpoint type inequalities as special cases of our main results.
\end{abstract}

MSC: Primary 26B30; secondary 26D07; 26D10

Keywords: Bounded functions; Fractional integrals; Ostrowski type inequalities; Midpoint type inequalities

\section{Introduction}

For well over a century, the study of various types of integral inequalities has been the focus of great attention by a number of mathematicians, interested both in pure and applied mathematics. One of the many fundamental mathematical discoveries of Ostrowski [1] is the following classical integral inequality associated with the differentiable mappings.

Theorem 1 Let $f:[a, b] \rightarrow \mathbb{R}$ be a differentiable mapping on $(a, b)$ whose derivative $f^{\prime}:(a, b) \rightarrow \mathbb{R}$ is bounded on $(a, b)$, i.e. $\left\|f^{\prime}\right\|_{\infty}:=\sup _{t \in(a, b)}\left|f^{\prime}(t)\right|<\infty$. Then we have the inequality

$$
\left|f(x)-\frac{1}{b-a} \int_{a}^{b} f(t) \mathrm{d} t\right| \leq\left[\frac{1}{4}+\frac{\left(x-\frac{a+b}{2}\right)^{2}}{(b-a)^{2}}\right](b-a)\left\|f^{\prime}\right\|_{\infty}
$$

for all $x \in[a, b]$.

The constant $\frac{1}{4}$ is the best possible.

The overall structure of the study takes the form of five sections including Introduction. The remainder of this work is organized as follows: we first give the definition of RiemannLiouville fractional integrals and mention some work which focuses on Ostrowski inequality. In Sect. 2, we obtain some generalized identities for the twice partial differentiable functions. Using the equalities obtained in Sect. 2, we establish some Ostrowski type inequalities for the functions belong to $L_{\infty}$ in Sect. 3, also we prove Ostrowski type inequalities for the mappings belong to $L_{p}$ and $L_{1}$ in Sect. 4 and Sect. 5 , respectively.

(c) The Author(s) 2020. This article is licensed under a Creative Commons Attribution 4.0 International License, which permits use sharing, adaptation, distribution and reproduction in any medium or format, as long as you give appropriate credit to the original author(s) and the source, provide a link to the Creative Commons licence, and indicate if changes were made. The images or other third party material in this article are included in the article's Creative Commons licence, unless indicated otherwise in a credit line to the material. If material is not included in the article's Creative Commons licence and your intended use is not permitted by statutory regulation or exceeds the permitted use, you will need to obtain permission directly from the copyright holder. To view a copy of this licence, visit http://creativecommons.org/licenses/by/4.0/. 
Firstly, we give the definitions of Riemann-Liouville fractional integrals.

Definition 1 ([2]) Let $f \in L_{1}[a, b]$. The Riemann-Liouville integrals $J_{a_{+}}^{\alpha} f$ and $J_{b-}^{\alpha} f$ of order $\alpha>0$ with $a \geq 0$ are defined by

$$
J_{a+}^{\alpha} f(x)=\frac{1}{\Gamma(\alpha)} \int_{a}^{x}(x-t)^{\alpha-1} f(t) \mathrm{d} t, \quad x>a
$$

and

$$
J_{b-}^{\alpha} f(x)=\frac{1}{\Gamma(\alpha)} \int_{x}^{b}(t-x)^{\alpha-1} f(t) \mathrm{d} t, \quad x<b
$$

respectively. Here, $\Gamma(\alpha)$ is the Gamma function and $J_{a+}^{0} f(x)=J_{b-}^{0} f(x)=f(x)$.

Now, we give the definitions Riemann-Liouville fractional integrals of two variable functions:

Definition 2 ([3]) Let $f \in L_{1}([a, b] \times[c, d])$. The Riemann-Liouville fractional integrals $J_{a+, c_{+},}^{\alpha, \beta} J_{a+, d-}^{\alpha, \beta}, J_{b-, c_{+}}^{\alpha, \beta}$ and $J_{b-, d_{-}}^{\alpha, \beta}$ are defined by

$$
\begin{array}{ll}
J_{a+, c+}^{\alpha, \beta} f(x, y)=\frac{1}{\Gamma(\alpha) \Gamma(\beta)} \int_{a}^{x} \int_{c}^{y}(x-t)^{\alpha-1}(y-s)^{\beta-1} f(t, s) \mathrm{d} s \mathrm{~d} t, & x>a, y>c, \\
J_{a+, d-}^{\alpha, \beta} f(x, y)=\frac{1}{\Gamma(\alpha) \Gamma(\beta)} \int_{a}^{x} \int_{y}^{d}(x-t)^{\alpha-1}(s-y)^{\beta-1} f(t, s) \mathrm{d} s \mathrm{~d} t, & x>a, y<d, \\
J_{b-,+c}^{\alpha, \beta} f(x, y)=\frac{1}{\Gamma(\alpha) \Gamma(\beta)} \int_{x}^{b} \int_{c}^{y}(t-x)^{\alpha-1}(y-s)^{\beta-1} f(t, s) \mathrm{d} s \mathrm{~d} t, & x<b, y>c,
\end{array}
$$

and

$$
J_{b-, d}^{\alpha, \beta} f(x, y)=\frac{1}{\Gamma(\alpha) \Gamma(\beta)} \int_{x}^{b} \int_{y}^{d}(t-x)^{\alpha-1}(s-y)^{\beta-1} f(t, s) \mathrm{d} s \mathrm{~d} t, \quad x<b, y<d .
$$

Ostrowski inequalities for fractional integrals of two variable functions are obtained in [4]. There are several papers on fractional Ostrowski type inequalities for one or two variable functions, you can find some of them in Refs. [5-22].

\section{Some identities for double integrals}

Some equalities including Riemann-Liouville fractional integrals of two variable functions are established in this section. These identities will be used to prove the inequalities developed throughout this study.

Firstly, we define the following functions which will be used frequently:

$$
\begin{aligned}
& M_{\alpha}(a, b ; x):=\frac{(x-a)^{\alpha}+(b-x)^{\alpha}}{\Gamma(\alpha+1)}, \\
& N_{\beta}(c, d ; y):=\frac{(y-c)^{\beta}+(d-y)^{\beta}}{\Gamma(\beta+1)},
\end{aligned}
$$

for $(x, y) \in \Lambda:=[a, b] \times[c, d]$.

Now we prove the following equalities. 
Lemma 1 Let $: \Lambda \rightarrow \mathbb{R}$ be an absolutely continuous function such that the partial derivative of order 2 exists and is continuous on $\Lambda$ in $\mathbb{R}^{2}$. Then, for any $(x, y) \in \Lambda$, we have

$$
\begin{aligned}
& \frac{1}{\Gamma(\alpha) \Gamma(\beta)} \int_{a}^{b} \int_{c}^{d} \Omega(x, t, y, s)\left[\int_{x}^{t} \int_{y}^{s} \frac{\partial^{2} f(\varsigma, \tau)}{\partial \varsigma \partial \tau} \mathrm{d} \tau \mathrm{d} \varsigma\right] \mathrm{d} s \mathrm{~d} t \\
&=J_{a+, c+}^{\alpha, \beta} f(x, y)+J_{a+, d}^{\alpha, \beta} f(x, y)+J_{b-, c+}^{\alpha, \beta} f(x, y)+J_{b-, d-}^{\alpha, \beta} f(x, y) \\
& \quad-N_{\beta}(c, d ; y)\left[J_{a+}^{\alpha} f(x, y)+J_{b-}^{\alpha} f(x, y)\right] \\
& \quad-M_{\alpha}(a, b ; x)\left[J_{c+}^{\beta} f(x, y)+J_{d-,}^{\beta} f(x, y)\right] \\
& \quad+M_{\alpha}(a, b ; x) N_{\beta}(c, d ; y) f(x, y) \\
&=: \mathcal{G}_{1}(x, y ; a, b, c, d),
\end{aligned}
$$

where $\Omega(x, t, y, s)$ is defined by

$$
\begin{aligned}
& \Omega(x, t, y, s) \\
& := \begin{cases}(x-t)^{\alpha-1}(y-s)^{\beta-1}, & a \leq t<x \text { and } c \leq s<y, \\
(x-t)^{\alpha-1}(s-y)^{\beta-1}, & a \leq t<x \text { and } y \leq s \leq d, \\
(t-x)^{\alpha-1}(y-s)^{\beta-1}, & x \leq t \leq b \text { and } c \leq s<y, \\
(t-x)^{\alpha-1}(s-y)^{\beta-1}, & x \leq t \leq b \text { and } y \leq s \leq d .\end{cases}
\end{aligned}
$$

Proof It is easy to see that

$$
\begin{aligned}
& \int_{x}^{t} \int_{y}^{s} \frac{\partial^{2} f(\varsigma, \tau)}{\partial \varsigma \partial \tau} \mathrm{d} \tau \mathrm{d} \varsigma \\
& \quad=f(t, s)-f(t, y)-f(x, s)+f(x, y) \\
& \quad=: F(x, t, y, s) .
\end{aligned}
$$

By the above equality and the definition of $\Omega(x, t, y, s)$, we get

$$
\begin{gathered}
\frac{1}{\Gamma(\alpha) \Gamma(\beta)} \int_{a}^{b} \int_{c}^{d} Q(x, t, y, s)\left[\int_{x}^{t} \int_{y}^{s} \frac{\partial^{2} f(\varsigma, \tau)}{\partial \varsigma \partial \tau} \mathrm{d} \tau \mathrm{d} \varsigma\right] \mathrm{d} s \mathrm{~d} t \\
=\frac{1}{\Gamma(\alpha) \Gamma(\beta)} \int_{a}^{x} \int_{c}^{y}(x-t)^{\alpha-1}(y-s)^{\beta-1} F(x, t, y, s) \mathrm{d} s \mathrm{~d} t \\
\quad+\frac{1}{\Gamma(\alpha) \Gamma(\beta)} \int_{a}^{x} \int_{y}^{d}(x-t)^{\alpha-1}(s-y)^{\beta-1} F(x, t, y, s) \mathrm{d} s \mathrm{~d} t \\
\quad+\frac{1}{\Gamma(\alpha) \Gamma(\beta)} \int_{x}^{b} \int_{c}^{y}(t-x)^{\alpha-1}(y-s)^{\beta-1} F(x, t, y, s) \mathrm{d} s \mathrm{~d} t \\
\quad+\frac{1}{\Gamma(\alpha) \Gamma(\beta)} \int_{x}^{b} \int_{y}^{d}(t-x)^{\alpha-1}(s-y)^{\beta-1} F(x, t, y, s) \mathrm{d} s \mathrm{~d} t .
\end{gathered}
$$


Applying the fundamental integral rules for the first integral in the right side of the above identity, we find that

$$
\begin{aligned}
& \frac{1}{\Gamma(\alpha) \Gamma(\beta)} \int_{a}^{x} \int_{c}^{y}(x-t)^{\alpha-1}(y-s)^{\beta-1} F(x, t, y, s) \mathrm{d} s \mathrm{~d} t \\
& =J_{a+, c+}^{\alpha, \beta} f(x, y)-\frac{(y-c)^{\beta}}{\Gamma(\beta+1)} J_{a+}^{\alpha} f(x, y) \\
& \quad-\frac{(x-a)^{\alpha}}{\Gamma(\alpha+1)} J_{c+}^{\beta} f(x, y)+\frac{(x-a)^{\alpha}(y-c)^{\beta}}{\Gamma(\alpha+1) \Gamma(\beta+1)} f(x, y) .
\end{aligned}
$$

Adding the resulting identities after calculating the other integrals, then the desired equality (2.1) can be attained.

Lemma 2 Suppose that all the assumptions of the Lemma 1 hold. Then, for any $(x, y) \in \Lambda$, we have

$$
\begin{aligned}
& \frac{1}{\Gamma(\alpha) \Gamma(\beta)} \int_{a}^{b} \int_{c}^{d} \Omega(t, s)\left[\int_{x}^{t} \int_{y}^{s} \frac{\partial^{2} f(\varsigma, \tau)}{\partial \varsigma \partial \tau} \mathrm{d} \tau \mathrm{d} \varsigma\right] \mathrm{d} s \mathrm{~d} t \\
&=J_{x+, y+}^{\alpha, \beta} f(b, d)+J_{x+, y-}^{\alpha, \beta} f(b, c)+J_{x-, y+}^{\alpha, \beta} f(a, d)+J_{x-, y-}^{\alpha, \beta} f(a, c) \\
& \quad-N_{\beta}(c, d ; y)\left[J_{x+}^{\alpha} f(b, y)+J_{x-}^{\alpha} f(a, y)\right] \\
& \quad-M_{\alpha}(a, b ; x)\left[J_{y+}^{\beta} f(x, d)+J_{y-}^{\beta} f(x, c)\right] \\
& \quad+M_{\alpha}(a, b ; x) N_{\beta}(c, d ; y) f(x, y) \\
&=: \mathcal{G}_{2}(x, y ; a, b, c, d),
\end{aligned}
$$

where $\Omega(t, s)$ is defined by

$$
\begin{aligned}
& \Omega(t, s) \\
& := \begin{cases}(t-a)^{\alpha-1}(s-c)^{\beta-1}, & a \leq t<x \text { and } c \leq s<y, \\
(t-a)^{\alpha-1}(d-s)^{\beta-1}, & a \leq t<x \text { and } y \leq s \leq d, \\
(b-t)^{\alpha-1}(s-c)^{\beta-1}, & x \leq t \leq b \text { and } c \leq s<y, \\
(b-t)^{\alpha-1}(d-s)^{\beta-1}, & x \leq t \leq b \text { and } y \leq s \leq d .\end{cases}
\end{aligned}
$$

Proof The proof of the equality (2.4) follows the same lines as the proof of Lemma 1.

Lemma 3 Suppose that all the assumptions of the Lemma 1 hold. Then, for any $(x, y) \in \Lambda$, we have

$$
\begin{aligned}
& \frac{1}{4 \Gamma(\alpha) \Gamma(\beta)}\left\{\int_{a}^{b} \int_{c}^{d}\left[(t-a)^{\alpha-1}+(b-t)^{\alpha-1}\right]\right. \\
& \left.\times\left[(s-c)^{\beta-1}+(d-s)^{\beta-1}\right]\left[\int_{x}^{t} \int_{y}^{s} \frac{\partial^{2} f(\varsigma, \tau)}{\partial \varsigma \partial \tau} \mathrm{d} \tau \mathrm{d} \varsigma\right] \mathrm{d} s \mathrm{~d} t\right\} \\
& =\frac{J_{b-, d-}^{\alpha, \beta} f(a, c)+J_{b-,+,}^{\alpha, \beta} f(a, d)+J_{a+, d-}^{\alpha, \beta} f(b, c)+J_{a_{+}, c+-}^{\alpha, \beta} f(b, d)}{4}
\end{aligned}
$$




$$
\begin{aligned}
& -\frac{1}{2} \frac{(d-c)^{\beta}}{\Gamma(\beta+1)}\left[J_{b-}^{\alpha} f(a, y)+J_{a_{+}}^{\alpha} f(b, y)\right] \\
& -\frac{1}{2} \frac{(b-a)^{\alpha}}{\Gamma(\alpha+1)}\left[J_{d-}^{\beta} f(x, c)+J_{c+}^{\beta} f(x, d)\right]+\frac{(b-a)^{\alpha}(d-c)^{\beta}}{\Gamma(\alpha+1) \Gamma(\beta+1)} f(x, y) \\
= & \mathcal{G}_{3}(x, y ; a, b, c, d) .
\end{aligned}
$$

Proof If we handle integral of the first expression in left side of (2.6), from the equality (2.3), then we have

$$
\begin{aligned}
& \frac{1}{\Gamma(\alpha) \Gamma(\beta)} \int_{a}^{b} \int_{c}^{d}(t-a)^{\alpha-1}(y-c)^{\beta-1} F(x, t, y, s) \mathrm{d} s \mathrm{~d} t \\
& =J_{b-, d-}^{\alpha, \beta} f(a, c)-\frac{(d-c)^{\beta}}{\Gamma(\beta+1)} J_{b-}^{\alpha} f(a, y) \\
& \quad-\frac{(b-a)^{\alpha}}{\Gamma(\alpha+1)} J_{d-}^{\beta} f(x, c)+\frac{(b-a)^{\alpha}(d-c)^{\beta}}{\Gamma(\alpha+1) \Gamma(\beta+1)} f(x, y) .
\end{aligned}
$$

Adding the resulting identities side by side after the other expressions have been similarly examined, the required equality can be easily derived.

\section{The case when $f_{5 \tau} \in L_{\infty}(\Lambda)$}

In this section, we observe some double integral inequalities involving Riemann-Liouville fractional expressions by considering identities given in the previous section and the functions that are element of $L_{\infty}$.

Theorem 2 Let $f: \Lambda \rightarrow \mathbb{R}$ be an absolutely continuous function such that the partial derivative of order 2 exists and is bounded, i.e.,

$$
\left\|f_{\varsigma \tau}\right\|_{\infty}=\sup _{(\varsigma, \tau) \in(a, b) \times(c, d)}\left|\frac{\partial^{2} f(\varsigma, \tau)}{\partial \varsigma \partial \tau}\right|<\infty
$$

for all $(\varsigma, \tau) \in \Lambda$. Then one has

$$
\left|\mathcal{G}_{1}(x, y ; a, b, c, d)\right| \leq M_{\alpha+1}(a, b ; x) N_{\beta+1}(c, d ; y)\left\|f_{\varsigma \tau}\right\|_{\infty}
$$

for all $(x, y) \in \Lambda$.

Proof Taking the absolute value of both sides of the equality (2.1), because $f_{\varsigma \tau}$ is a bounded function on $\Lambda$, it follows that

$$
\begin{aligned}
& \left|\mathcal{G}_{1}(x, y ; a, b, c, d)\right| \\
& \quad \leq\left\|f_{\varsigma \tau}\right\|_{\infty} \frac{1}{\Gamma(\alpha) \Gamma(\beta)} \int_{a}^{b} \int_{c}^{d}|\Omega(x, t, y, s)||t-x||s-y| \mathrm{d} s \mathrm{~d} t .
\end{aligned}
$$

Using the definition of $\Omega(x, t, y, s)$ and elementary analysis operations, the desired result can be easily obtained. 
Corollary 1 If we choose $x=\frac{a+b}{2}$ and $y=\frac{c+d}{2}$ in (3.1), then we have the midpoint inequality

$$
\begin{aligned}
\mid J_{a+, c+}^{\alpha, \beta} f\left(\frac{a+b}{2}, \frac{c+d}{2}\right)+J_{a+, d-}^{\alpha, \beta} f\left(\frac{a+b}{2}, \frac{c+d}{2}\right) \\
+J_{b-, c+}^{\alpha, \beta} f\left(\frac{a+b}{2}, \frac{c+d}{2}\right)+J_{b-, d-}^{\alpha, \beta} f\left(\frac{a+b}{2}, \frac{c+d}{2}\right) \\
\quad-\frac{(d-c)^{\beta}}{2^{\beta-1} \Gamma(\beta+1)}\left[J_{a+}^{\alpha} f\left(\frac{a+b}{2}, \frac{c+d}{2}\right)+J_{b-}^{\alpha} f\left(\frac{a+b}{2}, \frac{c+d}{2}\right)\right] \\
\quad-\frac{(b-a)^{\alpha}}{2^{\alpha-1} \Gamma(\alpha+1)}\left[J_{c+}^{\beta} f\left(\frac{a+b}{2}, \frac{c+d}{2}\right)+J_{d-}^{\beta} f\left(\frac{a+b}{2}, \frac{c+d}{2}\right)\right] \\
\quad+\frac{(b-a)^{\alpha}(d-c)^{\beta}}{2^{\alpha+\beta-2} \Gamma(\alpha+1) \Gamma(\beta+1)} f\left(\frac{a+b}{2}, \frac{c+d}{2}\right) \mid \\
\leq \frac{(b-a)^{\alpha+1}(d-c)^{\beta+1}}{2^{\alpha+\beta} \Gamma(\alpha+2) \Gamma(\beta+2)}\left\|f_{\varsigma \tau}\right\|_{\infty} .
\end{aligned}
$$

Theorem 3 Suppose that all the assumptions of Theorem 2 hold. Then we have

$$
\left|\mathcal{G}_{2}(x, y ; a, b, c, d)\right| \leq M_{\alpha+1}(a, b ; x) N_{\beta+1}(c, d ; y)\left\|f_{\varsigma \tau}\right\|_{\infty}
$$

for any $(x, y) \in \Lambda$.

Proof This proof follows the same strategy which was used in the proof of Theorem 2 by considering the equality (2.4).

Corollary 2 With the assumption of Theorem 3, one has the midpoint type inequality

$$
\begin{aligned}
& \mid J_{\frac{a+b}{2}+, \frac{c+d}{2}+}^{\alpha, \beta} f(b, d)+J_{\frac{a+b}{2}+, \frac{c+d}{2}-}^{\alpha, \beta} f(b, c)+J_{\frac{a+b}{2}-\frac{c+d}{2}+}^{\alpha, \beta} f(a, d)+J_{\frac{a+b}{2}-, \frac{c+d}{2}-}^{\alpha, \beta} f(a, c) \\
& \quad-\frac{(d-c)^{\beta}}{2^{\beta-1} \Gamma(\beta+1)}\left[J_{\frac{a+b}{2}+}^{\alpha} f\left(b, \frac{c+d}{2}\right)+J_{\frac{a+b}{2}}^{\alpha} f\left(a, \frac{c+d}{2}\right)\right] \\
& \quad-\frac{(b-a)^{\alpha}}{2^{\alpha-1} \Gamma(\alpha+1)}\left[J_{\frac{c+d}{2}+,}^{\beta} f\left(\frac{a+b}{2}, d\right)+J_{\frac{c+d}{2}-,}^{\beta} f\left(\frac{a+b}{2}, c\right)\right] \\
& \quad+\frac{(b-a)^{\alpha}(d-c)^{\beta}}{2^{\alpha+\beta-2} \Gamma(\alpha+1) \Gamma(\beta+1)} f\left(\frac{a+b}{2}, \frac{c+d}{2}\right) \mid \\
& \leq \quad \frac{(b-a)^{\alpha+1}(d-c)^{\beta+1}}{2^{\alpha+\beta} \Gamma(\alpha+2) \Gamma(\beta+2)}\left\|f_{\varsigma \tau}\right\|_{\infty} .
\end{aligned}
$$

Theorem 4 Suppose that all the assumptions of Theorem 2 hold. Then, for all $(x, y) \in \Lambda$, one has

$$
\begin{aligned}
&\left|\mathcal{G}_{3}(x, y ; a, b, c, d)\right| \\
& \leq \frac{1}{4 \Gamma(\alpha) \Gamma(\beta)}\left[R_{\alpha}(x ; a, b)+S_{\alpha}(x ; a, b)\right] \\
& \quad \times\left[R_{\beta}(y ; c, d)+S_{\beta}(y ; c, d)\right]\left\|f_{\varsigma \tau}\right\|_{\infty},
\end{aligned}
$$


where

$$
R_{\lambda}(\xi ; u, v)=2 \frac{(\xi-u)^{\lambda+1}}{\lambda(\lambda+1)}+(v-u)^{\lambda}\left(\frac{v-u}{\lambda+1}-\frac{\xi-u}{\lambda}\right)
$$

and

$$
S_{\lambda}(\xi ; u, v)=2 \frac{(v-\xi)^{\lambda+1}}{\lambda(\lambda+1)}+(v-u)^{\lambda}\left(\frac{v-u}{\lambda+1}-\frac{v-\xi}{\lambda}\right)
$$

Proof Should we take the absolute value of (2.6), from the modulus property of the integral, then we have the inequality

$$
\begin{aligned}
& \left|\mathcal{G}_{3}(x, y ; a, b, c, d)\right| \\
& \quad \leq \mid f_{\varsigma \tau} \|_{\infty} \frac{1}{4} \frac{1}{\Gamma(\alpha) \Gamma(\beta)}\left\{\int_{a}^{b} \int_{c}^{d}\left[(t-a)^{\alpha-1}+(b-t)^{\alpha-1}\right]\right. \\
& \left.\quad \times\left[(s-c)^{\beta-1}+(d-s)^{\beta-1}\right]|t-x||s-y| \mathrm{d} s \mathrm{~d} t\right\} .
\end{aligned}
$$

Calculating the double integral given in the right side of (3.4), the desired inequality (3.3) can be easily obtained.

Corollary 3 Suppose that all the assumptions of Theorem 4 hold. If we choose $x=\frac{a+b}{2}$ and $y=\frac{c+d}{2}$, then we have

$$
\begin{aligned}
& \mid \frac{J_{b-, d-}^{\alpha, \beta} f(a, c)+J_{b-, c+}^{\alpha, \beta} f(a, d)+J_{a+, d}^{\alpha, \beta} f(b, c)+J_{a+, c+-}^{\alpha, \beta} f(b, d)}{4} \\
& \quad-\frac{1}{2} \frac{(d-c)^{\beta}}{\Gamma(\beta+1)}\left[J_{b-}^{\alpha} f\left(a, \frac{c+d}{2}\right)+J_{a+}^{\alpha} f\left(b, \frac{c+d}{2}\right)\right] \\
& \quad-\frac{1}{2} \frac{(b-a)^{\alpha}}{\Gamma(\alpha+1)}\left[J_{d-}^{\beta} f\left(\frac{a+b}{2}, c\right)+J_{c+}^{\beta} f\left(\frac{a+b}{2}, d\right)\right] \\
& \quad+\frac{(b-a)^{\alpha}(d-c)^{\beta}}{\Gamma(\alpha+1) \Gamma(\beta+1)} f\left(\frac{a+b}{2}, \frac{c+d}{2}\right) \mid \\
& \leq \frac{(b-a)^{\alpha+1}(d-c)^{\beta+1}}{\Gamma(\alpha+2) \Gamma(\beta+2)}\left[\frac{1}{2^{\alpha}}+\frac{\alpha-1}{2}\right]\left[\frac{1}{2^{\beta}}+\frac{\beta-1}{2}\right]\left\|f_{\varsigma \tau}\right\|_{\infty} .
\end{aligned}
$$

\section{The case when $f_{5 \tau} \in L_{p}(\Lambda)$}

In this section, we examine how to obtain inequalities for mappings which are elements of the space $L_{p}$.

Theorem 5 Let $f: \Lambda \rightarrow \mathbb{R}$ be an absolutely continuous function such that the partial derivative of order 2 exists on $(a, b) \times(c, d)$. If $\frac{\partial^{2} f(\zeta, \tau)}{\partial \zeta \partial \tau} \in L_{p}(\Lambda)$ for $p>1$ with $\frac{1}{p}+\frac{1}{q}=1$, i.e.,

$$
\left\|f_{\varsigma \tau}\right\|_{p}=\left(\int_{a}^{b} \int_{c}^{d}\left|\frac{\partial^{2} f(\varsigma, \tau)}{\partial \varsigma \partial \tau}\right|^{p} \mathrm{~d} \tau \mathrm{d} \varsigma\right)^{\frac{1}{p}}<\infty
$$


for all $(\varsigma, \tau) \in \Lambda$, then we have the inequality

$$
\begin{aligned}
& \left|\mathcal{G}_{1}(x, y ; a, b, c, d)\right| \\
& \quad \leq \frac{\left[(x-a)^{\alpha+\frac{1}{q}}+(b-x)^{\alpha+\frac{1}{q}}\right]\left[(y-c)^{\beta+\frac{1}{q}}+(d-y)^{\beta+\frac{1}{q}}\right]}{\Gamma(\alpha) \Gamma(\beta)(\alpha+1 / q)(\beta+1 / q)}\left\|f_{\varsigma \tau}\right\|_{p}
\end{aligned}
$$

for all $(x, y) \in \Lambda$.

Proof If we take the absolute value of both sides of the equality (2.1) and later apply the Hölder inequality, from the assumption of the function $f$, we have

$$
\begin{aligned}
& \Gamma(\alpha) \Gamma(\beta)\left|\mathcal{G}_{1}(x, y ; a, b, c, d)\right| \\
& \quad \leq \int_{a}^{b} \int_{c}^{d}|\Omega(x, t, y, s)|\left|\int_{x}^{t} \int_{y}^{s} \frac{\partial^{2} f(\varsigma, \tau)}{\partial \varsigma \partial \tau} \mathrm{d} \tau \mathrm{d} \varsigma\right| \mathrm{d} s \mathrm{~d} t \\
& \quad \leq\left.\left.\int_{a}^{b} \int_{c}^{d}|\Omega(x, t, y, s)||t-x|^{\frac{1}{q}}|s-y|^{\frac{1}{q}}\left|\int_{x}^{t} \int_{y}^{s}\right| \frac{\partial^{2} f(\varsigma, \tau)}{\partial \varsigma \partial \tau}\right|^{p} \mathrm{~d} \tau \mathrm{d} \varsigma\right|^{\frac{1}{p}} \mathrm{~d} s \mathrm{~d} t \\
& \quad=\int_{a}^{b} \int_{c}^{d}|\Omega(x, t, y, s)||t-x|^{\frac{1}{q}}|s-y|^{\frac{1}{q}}\left\|f_{\zeta \tau}\right\|_{[t, x] \times[s, y], p} \mathrm{~d} s \mathrm{~d} t \\
& \quad \leq\left\|f_{\varsigma \tau}\right\|_{p} \int_{a}^{b} \int_{c}^{d}|\Omega(x, t, y, s)||t-x|^{\frac{1}{q}}|s-y|^{\frac{1}{q}} \mathrm{~d} s \mathrm{~d} t .
\end{aligned}
$$

If the last integral of above expression is observed by considering the definition of $\Omega(x, t, y, s)$, then one attains the required inequality (4.1) which completes the proof.

Theorem 6 Assume that all the assumptions of Theorem 2 hold. If $\frac{\partial^{2} f(\varsigma, \tau)}{\partial \varsigma \partial \tau} \in L_{p}(\Lambda)$ for $p>1$ with $\frac{1}{p}+\frac{1}{q}=1$, then one has

$$
\begin{aligned}
&\left|\mathcal{G}_{2}(x, y ; a, b, c, d)\right| \\
& \leq \frac{[\Gamma(1+1 / q)]^{2}}{\Gamma(\alpha+1+1 / q) \Gamma(\beta+1+1 / q)}\left\|f_{\varsigma \tau}\right\|_{p} \\
& \quad \times\left[(x-a)^{\alpha+\frac{1}{q}}+(b-x)^{\alpha+\frac{1}{q}}\right]\left[(y-c)^{\beta+\frac{1}{q}}+(d-y)^{\beta+\frac{1}{q}}\right]
\end{aligned}
$$

for any $(x, y) \in \Lambda$.

Proof If similar methods to the proof of Theorem 5 are followed by taking into account the equality (2.4), because of the definition of $\Omega(t, s)$, then one has

$$
\begin{aligned}
\Gamma(\alpha) & \Gamma(\beta)\left|\mathcal{G}_{2}(x, y ; a, b, c, d)\right| \\
\leq & \left\|f_{\varsigma \tau}\right\|_{p} \int_{a}^{b} \int_{c}^{d}|\Omega(t, s)||t-x|^{\frac{1}{q}}|s-y|^{\frac{1}{q}} \mathrm{~d} s \mathrm{~d} t \\
= & \left\|f_{\varsigma \tau}\right\|_{p} \int_{a}^{x} \int_{c}^{y}(t-a)^{\alpha-1}(s-c)^{\beta-1}|t-x|^{\frac{1}{q}}|s-y|^{\frac{1}{q}} \mathrm{~d} s \mathrm{~d} t \\
& +\left\|f_{\varsigma \tau}\right\|_{p} \int_{a}^{x} \int_{y}^{d}(t-a)^{\alpha-1}(d-s)^{\beta-1}|t-x|^{\frac{1}{q}}|s-y|^{\frac{1}{q}} \mathrm{~d} s \mathrm{~d} t
\end{aligned}
$$




$$
\begin{aligned}
& +\left\|f_{\varsigma \tau}\right\|_{p} \int_{x}^{b} \int_{c}^{y}(b-t)^{\alpha-1}(s-c)^{\beta-1}|t-x|^{\frac{1}{q}}|s-y|^{\frac{1}{q}} \mathrm{~d} s \mathrm{~d} t \\
& +\left\|f_{\varsigma \tau}\right\|_{p} \int_{x}^{b} \int_{y}^{d}(b-t)^{\alpha-1}(d-s)^{\beta-1}|t-x|^{\frac{1}{q}}|s-y|^{\frac{1}{q}} \mathrm{~d} s \mathrm{~d} t .
\end{aligned}
$$

The above integrals can be readily calculated by utilizing the fact that

$$
\begin{aligned}
\int_{\lambda}^{\mu}(\xi-\lambda)^{\rho-1}(\mu-\xi)^{\sigma-1} \mathrm{~d} \xi & =(\mu-\lambda)^{\rho+\sigma-1} \int_{0}^{1} u^{\rho-1}(1-u)^{\sigma-1} \mathrm{~d} u \\
& =(\mu-\lambda)^{\rho+\sigma-1} B(\rho, \sigma),
\end{aligned}
$$

which is obtained by using the change of variable $\xi=(1-u) \lambda+u \mu$, and where $B(\cdot, \cdot)$ is Beta function. Hence, the proof is finished.

We also note that if we choose $x=\frac{a+b}{2}$ and $y=\frac{c+d}{2}$ in two inequalities presented in this section, then we reach new midpoint type results different from inequalities given in corollaries of the previous section.

\section{The case when $f_{5 \tau} \in L_{1}(\Lambda)$}

Now, we investigate how to results in the case when $f$ element of $L_{1}$.

Theorem 7 Let $f: \Lambda \rightarrow \mathbb{R}$ be an absolutely continuous function such that the partial derivative of order 2 exists on $(a, b) \times(c, d)$. If $\frac{\partial^{2} f(\varsigma, \tau)}{\partial \varsigma \partial \tau} \in L_{1}(\Lambda)$, i.e.,

$$
\left\|f_{\varsigma \tau}\right\|_{1}=\int_{a}^{b} \int_{c}^{d}\left|\frac{\partial^{2} f(\varsigma, \tau)}{\partial \varsigma \partial \tau}\right| \mathrm{d} \tau \mathrm{d} \varsigma<\infty
$$

for all $(\varsigma, \tau) \in \Lambda$, then we have the inequalities

$$
\left|\mathcal{G}_{1}(x, y ; a, b, c, d)\right| \leq M_{\alpha}(a, b ; x) N_{\beta}(c, d ; y)\left\|f_{\varsigma \tau}\right\|_{1}
$$

and

$$
\left|\mathcal{G}_{2}(x, y ; a, b, c, d)\right| \leq M_{\alpha}(a, b ; x) N_{\beta}(c, d ; y)\left\|f_{\varsigma \tau}\right\|_{1}
$$

for all $(x, y) \in \Lambda$.

Proof If we take the absolute value of both sides of the equality (2.1), due to the assumption of the function $f$, we have

$$
\begin{aligned}
& \left|\mathcal{G}_{1}(x, y ; a, b, c, d)\right| \\
& \quad \leq \frac{1}{\Gamma(\alpha) \Gamma(\beta)} \int_{a}^{b} \int_{c}^{d}|\Omega(x, t, y, s)|\left|\int_{x}^{t} \int_{y}^{s} \frac{\partial^{2} f(\varsigma, \tau)}{\partial \varsigma \partial \tau} \mathrm{d} \tau \mathrm{d} \varsigma\right| \mathrm{d} s \mathrm{~d} t \\
& \quad \leq\left\|f_{\varsigma \tau}\right\|_{1} \frac{1}{\Gamma(\alpha) \Gamma(\beta)} \int_{a}^{b} \int_{c}^{d}|\Omega(x, t, y, s)| \mathrm{d} s \mathrm{~d} t .
\end{aligned}
$$


Later, utilizing the definition of $\Omega(x, t, y, s)$, the desired inequality (5.1) can be readily attained. If we follow the same line as the proof of (5.1) by taking into account the equality (2.4), then we can also obtain the inequality (5.2). The proof is thus completed.

Theorem 8 Suppose that all the assumptions of Theorem 7 hold. If $\frac{\partial^{2} f(\varsigma, \tau)}{\partial \varsigma \partial \tau} \in L_{1}(\Lambda)$, then one has

$$
\left|\mathcal{G}_{3}(x, y ; a, b, c, d)\right| \leq \frac{(b-a)^{\alpha}(d-c)^{\beta}}{\Gamma(\alpha+1) \Gamma(\beta+1)}\left\|f_{\varsigma \tau}\right\|_{1}
$$

for all $(x, y) \in \Lambda$.

Proof Taking the absolute value of (2.6), from the modulus property of the integral, we find that

$$
\begin{aligned}
& \left|\mathcal{G}_{3}(x, y ; a, b, c, d)\right| \\
& \leq \frac{1}{4 \Gamma(\alpha) \Gamma(\beta)}\left\|f_{\varsigma \tau}\right\|_{1} \\
& \quad \times \int_{a}^{b} \int_{c}^{d}\left[(t-a)^{\alpha-1}+(b-t)^{\alpha-1}\right]\left[(s-c)^{\beta-1}+(d-s)^{\beta-1}\right] \mathrm{d} s \mathrm{~d} t .
\end{aligned}
$$

Calculating the above double integral, the required inequality (5.3) can be easily obtained.

\section{Acknowledgements}

This research is supported by Postdoctoral Fellowship from King Mongkut's University of Technology Thonburi (KMUTT), Bangkok, Thailand.

\section{Funding}

This research was funded by the Center of Excellence in Theoretical and Computational Science (TaCS-CoE), KMUTT.

Availability of data and materials

Not applicable.

\section{Competing interests}

The author declares that they have no competing interests.

\section{Authors' contributions}

The main idea of this paper was proposed by SE, HB, and PK. MZS and SI prepared the manuscript initially and performed all the steps of the proofs in this research. All authors read and approved the final manuscript.

\section{Author details}

'Department of Mathematics, Faculty of Science, Bartın University, Bartın, Turkey. ${ }^{2}$ Department of Mathematics, Faculty of Science and Arts, Düzce University, Düzce, Turkey. ${ }^{3}$ Center of Excellence in Theoretical and Computational Science (TaCS-CoE), Science Laboratory Building, Faculty of Science, King Mongkut's University of Technology Thonburi (KMUTT), Bangkok, Thailand. ${ }^{4}$ KMUTT Fixed Point Research Laboratory, KMUTT-Fixed Point Theory and Applications Research Group, SCL 802 Fixed Point Laboratory, Department of Mathematics, Faculty of Science, King Mongkut's University of Technology Thonburi (KMUTT), Bangkok, Thailand. ${ }^{5}$ Department of Medical Research, China Medical University Hospital, China Medical University, Taichung, Taiwan.

\section{Publisher's Note}

Springer Nature remains neutral with regard to jurisdictional claims in published maps and institutional affiliations.

Received: 4 December 2019 Accepted: 16 April 2020 Published online: 03 May 2020

\section{References}

1. Ostrowski, A.: Über die absolutabweichung einer differentiierbaren funktion von ihrem integralmittelwert. Comment. Math. Helv. 10, 226-227 (1937) 
2. Kilbas, A.A., Srivastava, H.M., Trujillo, J.J.: Theory and Applications of Fractional Differential Equations. North-Holland Mathematics Studies, vol. 204 (2006)

3. Sarıkaya, M.Z:: On the Hermite-Hadamard-type inequalities for co-ordinated convex function via fractional integrals. Integral Transforms Spec. Funct. 25(2), 134-147 (2014)

4. Latif, M.A., Dragomir, S., Matouk, A.: New inequalities of Ostrowski type for co-ordinated convex functions via fractional integrals. J. Fract. Calc. Appl. 2(1), 1-15 (2012)

5. Aglić Aljinović, A.: Montgomery identity and Ostrowski type inequalities for Riemann-Liouville fractional integral. J. Math. 2014 Article ID 503195 (2014)

6. Anastassiou, G., Hooshmandasl, M., Ghasemi, A., Moftakharzadeh, F.: Montgomery identities for fractional integrals and related fractional inequalities. JIPAM. J. Inequal. Pure Appl. Math. 10(4), 1-6 (2009)

7. Barnett, N.S., Dragomir, S.S.: An Ostrowski type inequality for double integrals and applications for cubature formulae. Soochow J. Math. 7(1), 109-114 (2001)

8. Dahmani, Z., et al.: On Minkowski and Hermite-Hadamard integral inequalities via fractional integration. Ann. Funct. Anal. 1(1), 51-58 (2010)

9. Dragomir, S.S.: Ostrowski type inequalities for Riemann-Liouville fractional integrals of absolutely continuous functions in terms of $\infty$-norms. RGMIA Res. Rep. Collect. 20, Article ID 49 (2017). Available online at https://rgmia.org/papers/v20/v20a49.pdf

10. Dragomir, S.S.: Ostrowski type inequalities for Riemann-Liouville fractional integrals of absolutely continuous functions in terms of $\infty$-norms. RGMIA Res. Rep. Collect. 20, Article ID 50 (2017). Available online at https://rgmia.org/papers/v20/v20a50.pdf

11. Dragomir, S.S., Barnett, N.S., Cerone, P.: An Ostrowski type inequality for double integrals in terms of $L_{p}$-norms and applications in numerical integration. Rev. Anal. Numér. Théor. Approx. 32(2), 161-169 (2003)

12. Dragomir, S.S.: Ostrowski and trapezoid type inequalities for generalized Riemann-Liouville fractional integrals of absolutely continuous functions with bounded derivatives. RGMIA Res. Rep. Collect. 20, 53 (2017)

13. Dragomir, S.S.: On some Ostrowski type inequalities for generalized Riemann-Liouville fractional integrals. RGMIA Res. Rep. Collect. 20,67 (2017)

14. Erden, S., Sarikaya, M.Z:: On the Hermite-Hadamard type and Ostrowski type inequalities for the co-ordinated convex functions. Palest. J. Math. 6(1) 257-270 (2017)

15. Guezane-Lakoud, A., Aissaoui, F.: New fractional inequalities of Ostrowski type. Transylv. J. Math. Mech. 5(2), 103-106 (2013)

16. Latif, M.A., Hussain, S., Dragomir, S.S.: New Ostrowski type inequalities for co-ordinated convex functions. TJMM 4(2), $125-136(2012)$

17. Mudassar, M., Siddidui, N., Iqbal, M.: On co-ordinated Ostrowski and Hadamard's type inequalities for convex functions. TJMM 9(1), 35-42 (2017)

18. Sarikaya, M.Z., Filiz, H.: Note on the Ostrowski type inequalities for fractional integrals. Vietnam J. Math. 42(2), 187-190 (2014)

19. Sarikaya, M.Z., Yildiz, M.K.: Generalization and Improvement of Ostrowski Type Inequalities. AIP Conference Proceedings, vol. 1991. AlP, New York (2018)

20. Sarikaya, M.Z., Budak, H., Yaldiz, H.: Some new Ostrowski type inequalities for co-ordinated convex functions. Turk. J. Anal. Number Theory 2(5), 176-182 (2014)

21. Set, E:: New inequalities of Ostrowski type for mappings whose derivatives are s-convex in the second sense via fractional integrals. Comput. Math. Appl. 63(7), 1147-1154 (2012)

22. Yaldız, H., Sarıkaya, M.Z., Dahmani, Z.: On the Hermite-Hadamard-Fejer-type inequalities for co-ordinated convex functions via fractional integrals. Int. J. Optim. Control Theor. Appl. 7(2), 205-215 (2017)

\section{Submit your manuscript to a SpringerOpen ${ }^{\circ}$ journal and benefit from:}

- Convenient online submission

- Rigorous peer review

- Open access: articles freely available online

- High visibility within the field

- Retaining the copyright to your article

Submit your next manuscript at $\gg$ springeropen.com 